\title{
Fourth- and fifth-order virial expansion of harmonically trapped fermions at unitarity
}

\author{
Y. Hou $\odot$, K. J. Morrell $\odot$, A. J. Czejdo $\odot$, and J. E. Drut $\odot$ \\ Department of Physics and Astronomy, University of North Carolina, Chapel Hill, North Carolina 27599, USA
}

(Received 7 May 2021; accepted 13 July 2021; published 28 July 2021)

\begin{abstract}
By generalizing our automated algebra approach from homogeneous space to harmonically trapped systems, we have calculated the fourth- and fifth-order virial coefficients of universal spin- $\frac{1}{2}$ fermions in the unitary limit, confined in an isotropic harmonic potential. We present results for said coefficients as a function of trapping frequency (or, equivalently, temperature), which compare favorably with previous Monte Carlo calculations (available only at fourth order) as well as with our previous estimates in the untrapped limit (high temperature, low frequency). In addition to the conventional coefficients, we provide estimates for the contributions from subspaces with varying polarization. We use our results for the virial coefficients, together with resummation techniques, to calculate the compressibility and spin susceptibility.
\end{abstract}

DOI: 10.1103/PhysRevResearch.3.033099

\section{INTRODUCTION}

At low enough temperatures, or high enough densities, matter invariably displays its quantum mechanical nature, first and foremost by virtue of quantum statistics (i.e., particles are ultimately bosonic or fermionic, at least in three spatial dimensions) but also due to interaction effects that may alter the nature of the equilibrium state. As the temperature is raised, these systems eventually undergo a quantum-classical crossover (QCC) in which interactions still play a role, but where quantum mechanical effects are slowly washed out by temperature fluctuations. This regime is especially interesting for strongly coupled matter, in particular in cases where a superfluid phase is present, as the behavior above the superfluid critical temperature (i.e., in the unordered phase) is still significantly affected by the interactions (e.g., inducing pairing correlations) but there are no obvious effective theory descriptions [1-10].

The QCC is governed by the so-called virial expansion (VE) [11], which breaks the quantum many-body problem into $n$-particle subspaces, captured in the so-called virial coefficients (see Ref. [12] for a review). For bulk thermodynamic quantities the virial coefficients are denoted by $b_{n}$, and their change due to interactions is $\Delta b_{n}$. The calculation of $\Delta b_{n}$ has a sparse history that started with $\Delta b_{2}$ in 1937, by Beth and Uhlenbeck [13] and remained largely quiet until the early 21 st century. On the theory side, this quiet period can be attributed to the well-known fact that the quantum two-body problem is considerably easier to solve (and to relate to twobody scattering properties) than its three- and higher-body counterparts. On the experimental side, these quantum virial

Published by the American Physical Society under the terms of the Creative Commons Attribution 4.0 International license. Further distribution of this work must maintain attribution to the author(s) and the published article's title, journal citation, and DOI. coefficients became increasingly relevant in the early 2000s with the rise of ultracold-atom experiments around the world and their ever-increasing ability to create, manipulate, and measure atomic clouds [14].

One of the most famous systems studied with ultracold atoms is the so-called unitary limit of the spin- $\frac{1}{2}$ Fermi gas [15], which represents a universal regime relevant for atomic and nuclear physics [16-21]. In this work we investigate the QCC of this universal regime using the VE up to fifth order for a system confined by a harmonic oscillator (HO) potential. Previous numerical work calculated $\Delta b_{3}$ [22-26], while Ref. [27] presented an essentially analytic calculation (naturally accompanied by a numerical evaluation of the resulting expressions at the end); notably, Ref. [28] calculated $\Delta b_{3}$ analytically for the unitary Bose gas, which requires three-body forces. Work on $\Delta b_{4}$ includes Refs. [29-32] (see also Refs. [33-35]). More recent work [36] studied analytic expressions in the so-called semiclassical approximation (previously implemented in a wide variety of situations [37,38], followed up by [39-42]), which uses a coarse discretization of imaginary time. On the experimental side, there have also been attempts to determine $\Delta b_{4}$ at unitarity in the untrapped limit, using measurements of the equation of state [43,44]. However, those analyses are numerically challenging because one must fit a fourth-order polynomial assuming higher-order contributions are small (which is not necessarily the case, as shown in Ref. [41]).

In this work we generalize the above calculations to include $\Delta b_{5}$ and go far beyond the semiclassical approximation, extrapolating to the continuous imaginary-time limit. While we restrict ourselves to the unitary limit in those extrapolations, we provide approximate analytic formulas that apply to arbitrary interaction strengths, trap frequency, and spatial dimension. For the purpose of this work, we have generalized the automated method of Ref. [41] to include a harmonic trapping potential. As we review below, the resulting approach is identical in spirit to the free-space case of Ref. [41] (namely, we automate the evaluation of canonical partition 
functions via Trotter-Suzuki factorization), but its implementation is very different in practice in the trapped case, which is best addressed in coordinate space. Crucially, the volume dependence of the canonical partition functions, which can be resolved analytically in the untrapped case, must be resolved numerically when the trap is turned on.

The remainder of this paper is organized as follows. The next section details the model Hamiltonian $\hat{H}$ and the virial expansion, whose coefficients are our main objective. Section III first explains how to obtain the virial coefficients from canonical partition functions (which is well known but we include for completeness) and then outlines the core of our method, namely, the use of a Trotter-Suzuki factorization of the quantum Boltzmann weight $e^{-\beta \hat{H}}$ into $N_{\tau}$ steps in order to access the $N$-particle transfer matrix, from which the $N$-body canonical partition function is obtained, thus completing the logical sequence that accesses the virial coefficients. Section IV presents our main results: approximate analytic expressions for the virial coefficients of trapped fermions in $d$ dimensions up to fifth order (for $N_{\tau}=1$ and 2, the latter reserved for Appendix B due to the length of the final expressions); virial coefficients of the unitary Fermi gas extrapolated to the $N_{\tau} \rightarrow \infty$ limit; and a simple application to the calculation of the compressibility and magnetic susceptibility. Finally, Sec. V presents our conclusions and future outlook.

\section{HAMILTONIAN AND VIRIAL EXPANSION}

In this work we focus on a system of harmonically trapped spin- $\frac{1}{2}$ fermions interacting via a short-range interaction. Thus, the Hamiltonian is $\hat{H}=\hat{T}+\hat{V}_{\text {ext }}+\hat{V}$, where

$$
\hat{T}=\sum_{s=\uparrow, \downarrow} \int d^{3} r \hat{\psi}_{s}^{\dagger}(\mathbf{r})\left(-\frac{\hbar \nabla^{2}}{2 m}\right) \hat{\psi}_{s}(\mathbf{r})
$$

is the kinetic energy operator,

$$
\hat{V}_{\text {ext }}=\int d^{3} r \frac{1}{2} m \omega^{2} \mathbf{r}^{2}\left[\hat{n}_{\uparrow}(\mathbf{r})+\hat{n}_{\downarrow}(\mathbf{r})\right]
$$

is the external potential energy operator, and

$$
\hat{V}=-g \int d^{3} r \hat{n}_{\uparrow}(\mathbf{r}) \hat{n}_{\downarrow}(\mathbf{r})
$$

is the interaction. Above, $m$ is the mass of the particles, $\omega$ is the isotropic harmonic trapping frequency, $g$ is the bare coupling, $\hat{n}_{s}(\mathbf{r})=\hat{\psi}_{s}^{\dagger}(\mathbf{r}) \hat{\psi}_{s}(\mathbf{r})$ is the particle density operator for spin- $s$ particles, and $\hat{\psi}_{s}^{\dagger}(\mathbf{r})$ and $\hat{\psi}_{s}(\mathbf{r})$ are, respectively, the creation and annihilation operators for particles of spin $s$ at position $\mathbf{r}$. We use units such that $\hbar=k_{B}=m=1$ from this point on. Naturally, the noninteracting piece $\hat{T}+\hat{V}_{\text {ext }}$ can be diagonalized exactly in the single-particle subspace of the Fock space, which leads to the HO basis we will refer to below. The contact interaction of Eq. (3) is singular in three spatial dimensions and must therefore be regularized and renormalized. To that end, we place the system on a spatial lattice of spacing $\ell$ and implicitly take the continuum limit by transforming spatial sums into integrals at the end. In the process, we renormalize by tuning the coupling so that the known two-body answer for the second-order virial coefficient is reproduced (see below).
The VE accesses thermodynamics by breaking down the calculation by particle number. Specifically, one expands the grand thermodynamic potential $\Omega$ in powers of the fugacity $z=\exp (\beta \mu)$ as

$$
-\beta \Omega=\ln \mathcal{Z}=Q_{1} \sum_{n=1}^{\infty} b_{n} z^{n},
$$

where $\beta$ is the inverse temperature, $Q_{1}$ is the single-particle partition function, and $b_{n}$ is the $n$ th-order virial coefficient. The $b_{n}$ capture, in a nonperturbative fashion, the contribution of the $n$-body problem to the full $\Omega$. Plugging in the definition of the grand-canonical partition function $\mathcal{Z}$, namely,

$$
\mathcal{Z}=\operatorname{tr}\left[e^{-\beta(\hat{H}-\mu \hat{N})}\right]=\sum_{N=0}^{\infty} z^{N} Q_{N}
$$

into Eq. (4) and expanding $\ln \mathcal{Z}$ in powers of $z$, the $b_{n}$ can be written in terms of the $N$-particle canonical partition functions

$$
Q_{N}=\operatorname{tr}_{N}\left[e^{-\beta \hat{H}}\right],
$$

where the trace is over the $N$-particle Hilbert space. As our system of interest contains particles of two different species, the $N$-body problem can be broken up into subspaces with $a$ particles of one type and $b$ particles of the other type, where $a+b=N$ (see below).

\section{COMPUTATIONAL FRAMEWORK}

\section{A. Obtaining virial coefficients from canonical partition functions.}

The interaction-induced change $\Delta b_{n}$, for $n=2,3,4,5$, is calculated as

$$
\begin{gathered}
\Delta b_{2}=\Delta b_{11}, \\
\Delta b_{3}=2 \Delta b_{21}, \\
\Delta b_{4}=2 \Delta b_{31}+\Delta b_{22}, \\
\Delta b_{5}=2 \Delta b_{41}+2 \Delta b_{32},
\end{gathered}
$$

where the subspace contributions are

$$
\begin{gathered}
\Delta b_{11}=\frac{\Delta Q_{11}}{Q_{1}}, \\
\Delta b_{21}=\frac{\Delta Q_{21}}{Q_{1}}-\frac{\Delta Q_{11}}{2}, \\
\Delta b_{31}=\frac{\Delta Q_{31}}{Q_{1}}-\frac{\Delta Q_{21}}{2}-\Delta Q_{11}\left(\frac{Q_{20}}{Q_{1}}-\frac{Q_{1}}{4}\right), \\
\Delta b_{22}=\frac{\Delta Q_{22}}{Q_{1}}-\Delta Q_{21}-\frac{\Delta\left(Q_{11}^{2}\right)}{2 Q_{1}}+\frac{\Delta Q_{11} Q_{1}}{2}, \\
\Delta b_{41}=\frac{\Delta Q_{41}}{Q_{1}}-\frac{\Delta Q_{31}}{2}-\Delta Q_{21}\left(\frac{Q_{20}}{Q_{1}}-\frac{Q_{1}}{4}\right) \\
-\Delta Q_{11}\left(\frac{Q_{30}}{Q_{1}}-Q_{20}+\frac{Q_{1}^{2}}{8}\right),
\end{gathered}
$$




$$
\begin{aligned}
\Delta b_{32}= & \frac{\Delta Q_{32}}{Q_{1}}-\frac{\Delta Q_{31}}{2}-\frac{\Delta Q_{22}}{2}-\frac{\Delta\left(Q_{21} Q_{11}\right)}{Q_{1}} \\
& -\Delta Q_{21}\left(\frac{Q_{20}}{Q_{1}}-\frac{3 Q_{1}}{4}\right)+\frac{\Delta\left(Q_{11}^{2}\right)}{2} \\
& +\Delta Q_{11}\left(Q_{20}-\frac{3 Q_{1}^{2}}{8}\right) .
\end{aligned}
$$

Here, $\Delta X$ represents the change in $X$ induced by the interactions and the $Q_{a b}$ are the canonical partition functions for $a$ particles of spin $\uparrow$ and $b$ particles of spin $\downarrow$. In the above expressions, the $\Delta b_{a b}$ are intensive quantities, whereas the $Q_{a b}$ themselves scale as $V^{a+b}$ where $V$ is the spatial volume (referring here to the scaling in the untrapped case). That property emphasizes the challenge in calculating $\Delta b_{a b}$ numerically: the delicate cancellations must be resolved among the various terms involving different $Q_{a b}$ 's, which becomes especially difficult in the $\beta \omega \rightarrow 0$ limit (we comment further on this technical issue below). It is for that reason that automated algebra methods are advocated here, where those cancellations can be resolved using arbitrary precision arithmetic, avoiding stochastic effects.

As the above equations refer to the change due to interactions, we note here for reference and completeness that the virial coefficients of noninteracting, harmonically trapped spin- $\frac{1}{2}$ fermions in $d$ spatial dimensions are given by

$$
b_{n}^{0}=\frac{(-1)^{n+1}}{n}\left(\frac{\sinh (\beta \omega / 2)}{\sinh (\beta \omega n / 2)}\right)^{d} .
$$

\section{B. Obtaining canonical partition functions from factorized transfer matrices}

To evaluate the canonical partition functions $Q_{a b}$, we implement a symmetric Suzuki-Trotter decomposition

$$
e^{-\beta \hat{H}}=\lim _{N_{\tau} \rightarrow \infty}\left[e^{-\frac{\tau}{2}\left(\hat{T}+\hat{V}_{\text {ext }}\right)} e^{-\tau \hat{V}} e^{-\frac{\tau}{2}\left(\hat{T}+\hat{V}_{\text {ext }}\right)}\right]^{N_{\tau}},
$$

where we split $\beta=\tau N_{\tau}$ into $N_{\tau}$ time steps. Thus,

$$
Q_{a b}=\lim _{N_{\tau} \rightarrow \infty} \operatorname{tr}_{a b}\left[e^{-\tau\left(\hat{T}+\hat{V}_{\text {ext }}\right)} e^{-\tau \hat{V}}\right]^{N_{\tau}},
$$

where the cyclic property of the trace was used. To proceed, we calculate the matrix elements of the factors inside the trace in coordinate space. For a single imaginary-time step, those matrix elements define the factorized transfer matrix $\mathcal{M}_{a b}$, for $a$ particles of spin $\uparrow$ and $b$ particles of spin $\downarrow$.

For example, by examining the $1+1$ subspace, i.e., $a=$ $b=1$, we obtain

$$
\begin{aligned}
{\left[\mathcal{M}_{11}\right]_{\mathbf{X}, \mathbf{Y}} } & =\left\langle\mathbf{X}\left|e^{-\tau\left(\hat{T}+\hat{V}_{\text {ext }}\right)} e^{-\tau \hat{V}}\right| \mathbf{Y}\right\rangle \\
& =\rho\left(\mathbf{x}_{1}, \mathbf{y}_{1}\right) \rho\left(\mathbf{x}_{2}, \mathbf{y}_{2}\right)\left[\mathbb{1}+C \delta\left(\mathbf{y}_{1}-\mathbf{y}_{2}\right)\right],
\end{aligned}
$$

where $\mathbf{X}=\left(\mathbf{x}_{1}, \mathbf{x}_{2}\right), \mathbf{Y}=\left(\mathbf{y}_{1}, \mathbf{y}_{2}\right), C=\left(e^{\tau g / \ell^{3}}-1\right) \ell^{3}$,

$$
\rho(\mathbf{x}, \mathbf{y})=\frac{1}{\lambda_{T}^{3}}\left[\frac{\beta \omega}{\sinh (\tau \omega)}\right]^{3 / 2} \exp \left[-\mathbf{Z}^{T} B \mathbf{Z}\right],
$$

with $\lambda_{T}=\sqrt{2 \pi \beta}, \mathbf{Z}^{T}=\left(\mathbf{x}^{T} / \lambda_{T}, \mathbf{y}^{T} / \lambda_{T}\right)$, and

$$
B=\frac{\pi \beta \omega}{\sinh (\tau \omega)}\left(\begin{array}{cc}
\cosh (\tau \omega) \mathbb{1} & -\mathbb{1} \\
-\mathbb{1} & \cosh (\tau \omega) \mathbb{1}
\end{array}\right),
$$

where $\mathbb{1}$ is a $3 \times 3$ unit matrix. The function $\rho(\mathbf{x}, \mathbf{y})$ in Eq. (21) is referred to as the Mehler kernel (see also Ref. [36] for further details) and is simply the coordinate representation of the transfer matrix of a single particle in the trapping potential. By taking the trace of $\mathcal{M}_{11}$, we obtain $Q_{11}$.

While the above example does not involve identical particles, for the cases that do (e.g., the $2+1$ subspace of the three-particle Hilbert space), the (anti)symmetrization can be carried out at the very end, i.e., after taking the $N_{\tau}$ th power of the distinguishable-particle transfer matrix $\mathcal{M}_{3}$. This property was already noted by Lee and Yang in $1959[45,46]$ and is a consequence of the fact that the operators involved do not change the particles' statistics. Thus, there is no need to use (anti)symmetrized intermediate states in the calculation, which greatly reduces the computational effort. All one needs is the distinguishable-particle transfer matrices $\mathcal{M}_{N}$. From there, the following identities are used to obtain the required canonical partition functions of the spin- $-\frac{1}{2}$ system:

$$
\begin{aligned}
& Q_{11}=\sum_{a b}\left[\mathcal{M}_{2}^{N_{\tau}}\right]_{a b, a b}, \\
& Q_{21}=\frac{1}{2 !} \sum_{a b c}\left\{\left[\mathcal{M}_{3}^{N_{\tau}}\right]_{a b c, a b c}-\left[\mathcal{M}_{3}^{N_{\tau}}\right]_{a b c, b a c}\right\} \\
& Q_{31}=\frac{1}{3 !} \sum_{a b c d}\left\{\left[\mathcal{M}_{4}^{N_{\tau}}\right]_{a b c d, a b c d}-3\left[\mathcal{M}_{4}^{N_{\tau}}\right]_{a b c d, b a c d}\right. \\
&\left.+2\left[\mathcal{M}_{4}^{N_{\tau}}\right]_{a b c d, b c a d}\right\} \\
& Q_{22}=\frac{1}{(2 !)^{2}} \sum_{a b c d}\left\{\left[\mathcal{M}_{4}^{N_{\tau}}\right]_{a b c d, a b c d}-2\left[\mathcal{M}_{4}^{N_{\tau}}\right]_{a b c d, a b d c}\right. \\
&\left.+\left[\mathcal{M}_{4}^{N_{\tau}}\right]_{a b c d, b a d c}\right\}, \\
& Q_{41}=\frac{1}{4 !} \sum_{a b c d e}\left\{\left[\mathcal{M}_{5}^{N_{\tau}}\right]_{a b c d e, a b c d e}-6\left[\mathcal{M}_{5}^{N_{\tau}}\right]_{a b c d e, a b d c e}\right. \\
&+3\left[\mathcal{M}_{5}^{N_{\tau}}\right]_{a b c d e, b a d c e}+8\left[\mathcal{M}_{5}^{N_{\tau}}\right]_{a b c d e, a c d b e} \\
&\left.+6\left[\mathcal{M}_{5}^{N_{\tau}}\right]_{a b c d e, b c d a e}\right\}, \\
& Q_{32}=\frac{1}{3 ! 2 !} \sum_{a b c d e}\left\{\left[\mathcal{M}_{5}^{N_{\tau}}\right]_{a b c d e, a b c d e}-3\left[\mathcal{M}_{5}^{N_{\tau}}\right]_{a b c d e, a c b d e}\right. \\
&+ 2\left[\mathcal{M}_{5}^{N_{\tau}}\right]_{a b c d e, b c a d e}-\left[\mathcal{M}_{5}^{N_{\tau}}\right]_{a b c d e, a b c e d} \\
&+\left.3\left[\mathcal{M}_{5}^{N_{\tau}}\right]_{a b c d e, a c b e d}-2\left[\mathcal{M}_{5}^{N_{\tau}}\right]_{a b c d e, b c a e d}\right\}
\end{aligned}
$$

In Appendix A we show the factorized form of the above transfer matrices $\mathcal{M}_{N}$. For our system of interest, these are written as products of the Mehler kernel of Eq. (21) and delta functions coming from the interaction (as in the example outlined above for $1+1$ particles). Notably, those expressions, as well as the above equations for $Q_{a b}$, remain unchanged as functionals of $\rho(\mathbf{x}, \mathbf{y})$ regardless of the form of the single-particle dispersion relation and trapping potential. Our formalism is thus straightforwardly generalized to a wide range of systems, as long as the analytic form of $\rho(\mathbf{x}, \mathbf{y})$ is known (see below).

Armed with the explicit $\mathcal{M}_{N}$ 's, we use automated algebra to symbolically expand $\left[\mathcal{M}_{N}\right]^{N_{\tau}}$ for varying $N_{\tau}$. For each $N_{\tau}$, one thus obtains high-order polynomials in the dimensionless 
bare coupling $C / \lambda_{T}^{3}$ (in three spatial dimensions). For the purposes of this work, we tune $C / \lambda_{T}^{3}$ such that the known $\Delta b_{2}$ for the unitary limit of the $3 \mathrm{D}$ Fermi gas is reproduced, as given by [12]

$$
\Delta b_{2}=\frac{1}{4} \operatorname{sech}\left(\frac{\beta \omega}{2}\right),
$$

which thus renormalizes the interaction for the desired value of $\beta \omega$. The value of $C / \lambda_{T}^{3}$ thus obtained is used to evaluate the higher-order virial coefficients $\Delta b_{a b}$. Once that is accomplished, we extrapolate to the large- $N_{\tau}$ limit.

In the above process, the coefficients of the various powers of $C / \lambda_{T}^{3}$ that appear in $Q_{a b}$ are given by multidimensional Gaussian integrals (coming from products of the Mehler kernel discussed above), which is the crucial property that enables precise numerical evaluation using modest-size determinants. The Gaussian integrals actually appear most naturally in the untrapped case, where one calculates in momentum space and where the volume dependence of the $Q_{a b}$ 's is resolved and canceled analytically to yield the intensive quantities $\Delta b_{n}$. For the trapped case, on the other hand, the Gaussian integrals appear when calculating in coordinate space, where the Mehler kernel allows a most direct evaluation. There is no well-defined volume dependence in this case; rather, the extensive behavior appears as terms that would diverge in the $\beta \omega \rightarrow 0$ limit but which cancel each other out exactly at finite $\beta \omega$. This cancellation is either verified exactly in fully analytic calculations (e.g., nothing diverges in the analytic expressions for $\Delta b_{n}$ shown below) or is resolved numerically (in the high- $N_{\tau}$ results we use for our extrapolations). Thus, although the factorization and automated algebra approach used here are identical in spirit to the free-space case of Ref. [41], their implementation is very different in practice and the trapped case involves the added challenge of resolving the cancellations mentioned above. Therefore, the technical aspects of this work represent a considerable advance and extension relative to those of Ref. [41].

As mentioned above, the formalism presented here remains unchanged for arbitrary systems, as long as the explicit form of the kernel $\rho(\mathbf{x}, \mathbf{y})$ is known analytically. However, the appearance of Gaussian integrals referred to in the previous paragraph is likely limited to the homogeneous and harmonically trapped cases. Still, it may be possible to expand a complicated $\rho(\mathbf{x}, \mathbf{y})$ in a basis of Gaussian functions. In that sense, our work may be considered as the first step in the characterization of more general systems.

\section{RESULTS}

\section{A. Approximate analytic expressions for $\Delta b_{n}$}

For $N_{\tau}=1,2$, we carry out calculations entirely analytically in which the coupling strength, the trapping frequency, and the spatial dimension appear as arbitrary variables (in principle, it is also possible to take this to even higher order, but the formulas become extremely long). The resulting formulas for $\Delta b_{3}$ and $\Delta b_{4}$ for $N_{\tau}=1$, first shown in Ref. [36], qualitatively (and in some parameter regions quantitatively) capture the behavior of $\Delta b_{n}$. These formulas are also useful as checks for codes that implement higher values of $N_{\tau}$. Here, we provide results broken down by subspace for up to five particles, shown in full detail in Appendix B. For $N_{\tau}=1$, one finds

$$
\begin{gathered}
\Delta b_{2}=\Delta b_{11}=\frac{1}{2} \frac{C}{\lambda_{T}^{d}}\left[\frac{\beta \omega}{2 \sinh (\beta \omega)}\right]^{d / 2}, \\
\Delta b_{21}=-\frac{\Delta b_{2}}{[2 \cosh (\beta \omega)+1]^{d / 2}}, \\
\Delta b_{31}=\frac{2^{-d / 2} \Delta b_{2}}{\cosh ^{d / 2}(\beta \omega)[2 \cosh (\beta \omega)+1]^{d / 2}},
\end{gathered}
$$

and

$$
\begin{aligned}
\Delta b_{22}= & \frac{2^{-3 d / 2} \Delta b_{2}}{\cosh ^{d / 2}(\beta \omega) \cosh ^{d}(\beta \omega / 2)} \\
& \times\left\{1+2^{d / 2} \Delta b_{2}\left[\cosh ^{d / 2}(\beta \omega)-2^{d / 2+1} \cosh ^{d}(\beta \omega / 2)\right]\right\} .
\end{aligned}
$$

From those formulas we learn that (as shown in Fig. 1), increasing $N_{\tau}$ does not immediately or necessarily improve the quality of the final answer; rather, the results could move away from the $N_{\tau} \rightarrow \infty$ limit before the asymptotic regime is reached, usually for $N_{\tau}>2$. Simply put, as $N_{\tau}$ is increased the results may worsen before they improve. Thus, it is important to investigate as large $N_{\tau}$ as possible, even if low values are qualitatively correct. In our automated calculations, we explored up to $N_{\tau}=20$ (for $\left.\Delta b_{21}\right), 16\left(\Delta b_{31}\right), 12\left(\Delta b_{22}\right), 12$ $\left(\Delta b_{41}\right)$, and $8\left(\Delta b_{32}\right)$, which we used to estimate the full $\Delta b_{3}$, $\Delta b_{4}$, and $\Delta b_{5}$, extrapolated to $N_{\tau} \rightarrow \infty$.

\section{B. Virial coefficients in the unitary limit}

As mentioned above, in our approach we calculate $\Delta b_{2}$ as a function of the bare coupling $C, \beta \omega$, and $N_{\tau}$, and renormalize by tuning $C$ to the known result in the unitary limit given by Eq. (29). Thus, the second-order VE is reproduced exactly by virtue of this renormalization condition, such that the line of constant physics is followed in the extrapolation to $N_{\tau} \rightarrow \infty$, for each $\beta \omega$ (see Supplemental Material of Ref. [41]).

In Fig. 1 we show our results for (a) $\Delta b_{3}$, (b) $\Delta b_{4}$, and (c) $\Delta b_{5}$ for a unitary Fermi gas in a harmonic trap as a function of $\beta \omega$. The error bars represent the uncertainty in the $N_{\tau} \rightarrow \infty$ extrapolation, given by the difference between the maximum and minimum predictions of polynomial extrapolation schemes (degrees 2 to 5 for $\Delta b_{3}$ and $\Delta b_{4}$, and degrees 2 and 3 for $\Delta b_{5}$, where the data are more limited; see Ref. [41]). Our results for $\Delta b_{3}$ are in superb agreement with the quantum Monte Carlo data of Ref. [29] as well as with the homogeneous-limit answer of Ref. [41]. Note that the homogeneous limit is related to the results shown here by

$$
\Delta b_{n}^{\mathrm{h}}=n^{3 / 2} \Delta b_{n}(\beta \omega \rightarrow 0)
$$

(see Refs. [12,22,23,47]).

The case of $\Delta b_{4}$ is less clear-cut: there is good agreement with Ref. [29] for $\beta \omega \geqslant 1$, but a clear difference remains at low frequencies. We return to this issue below. Finally, we predict $\Delta b_{5}$ as a function of $\beta \omega$, which to the best of our knowledge does not appear elsewhere in the literature. As the $\mathrm{HO}$ potential confines the system, it naturally increases its kinetic energy, effectively reducing the interaction effects. 

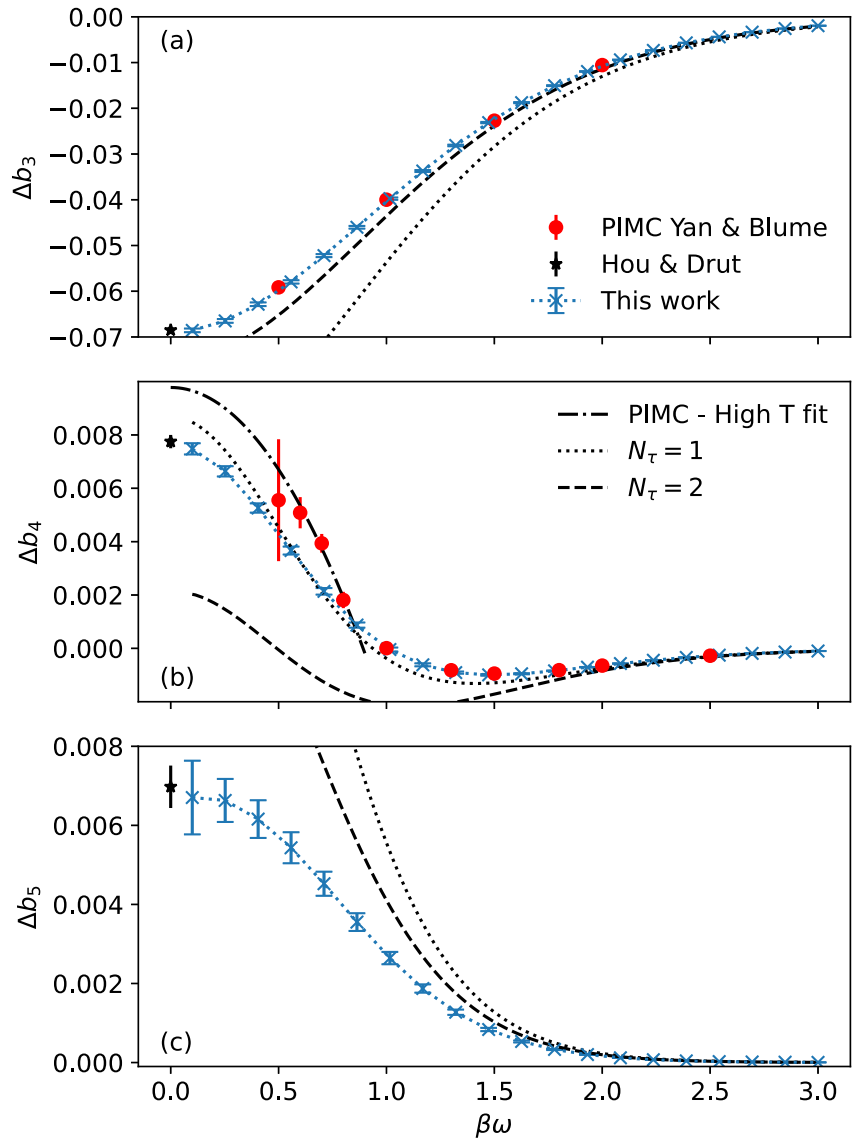

FIG. 1. (a) $\Delta b_{3}$, (b) $\Delta b_{4}$, and (c) $\Delta b_{5}$ as functions of $\beta \omega$, for a trapped unitary Fermi gas. Our results are shown with blue crosses and error bars, joined by a blue dotted line. The data by Yan and Blume from Ref. [29] appear as red circles for (a) $\Delta b_{3}$ and (b) $\Delta b_{4}$, in both cases with error bars. The dashed-dotted line in (b) shows a high-temperature fit to the data of Ref. [29]. Black stars with error bars show the results by Hou and Drut from Ref. [41] calculated in the homogeneous gas limit. The dotted (dashed) line shows the $N_{\tau}=$ $1\left(N_{\tau}=2\right)$ results given analytically in Appendix B. The latter show that, for $\Delta b_{3}$, increasing $N_{\tau}$ from 1 to 2 shows a clear improvement, whereas the case of $\Delta b_{4}$ is a cautionary tale: as $N_{\tau}$ goes from 1 to 2 , the results move away from our final answer (blue crosses). In fact, it is not until $N_{\tau}=5$ that $\Delta b_{4}$ reaches the asymptotic regime one can use for extrapolation. Reference [30] presented a large- $\beta \omega$ asymptotic formula for $\Delta b_{n}$, but its validity is well outside the $0<$ $\beta \omega<3$ region studied here.

This suggests that, for a given interaction strength, the VE should enjoy better convergence properties when a trapping potential is turned on (as argued also in Ref. [12]). Indeed, although our results indicate that $\Delta b_{4} \simeq \Delta b_{5}$ and, moreover, for $0.3<\beta \omega<1.4$ we find $\Delta b_{5}>\left|\Delta b_{4}\right|$, we also find that $\left|\Delta b_{2}\right| \gg\left|\Delta b_{3}\right| \gg\left|\Delta b_{4}\right|$.

To better understand the differences in $\Delta b_{4}$ between our results and Ref. [29], we plot in Fig. 2(a) the subspace contributions $\Delta b_{31}$ and $\Delta b_{22}$. As pointed out in Ref. [29], these contributions partially cancel each other out, leading to the observed increased uncertainty in the final answer. Clearly, the largest differences arise in the determination of $\Delta b_{22}$, which
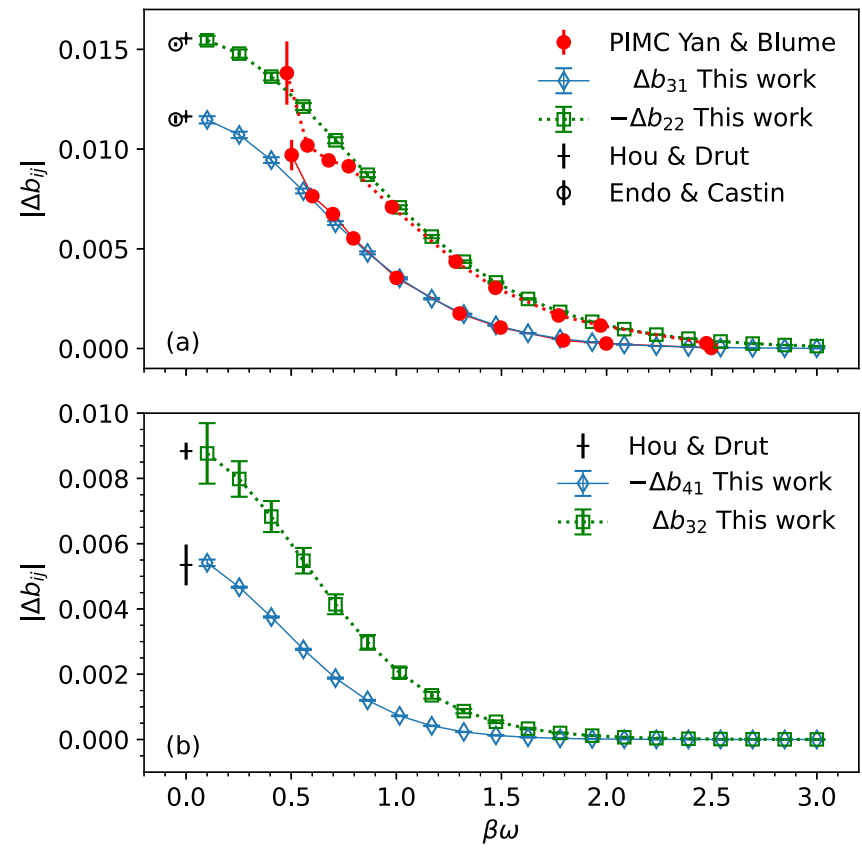

FIG. 2. (a) $\Delta b_{31}$ (blue diamonds) and $-\Delta b_{22}$ (green squares) as functions of $\beta \omega$, compared with the path-integral Monte Carlo (PIMC) results of Ref. [29] (red circles joined by solid line for $\Delta b_{31}$ and joined by a dotted line for $\Delta b_{22}$ ). The black crosses show the results at $\beta \omega=0$ from Ref. [41], and the black circle shows the corresponding results from Ref. [26]. (b) $-\Delta b_{41}$ (blue diamonds) and $\Delta b_{32}$ (green squares) as functions of $\beta \omega$. The black cross shows the results at $\beta \omega=0$ from Ref. [41].

is not unexpected as a contact interaction in that subspace is less susceptible to Pauli blocking than $\Delta b_{31}$.

Figure 2(b) shows our results for $\Delta b_{41}$ and $\Delta b_{32}$, whose behavior parallels $\Delta b_{31}$ and $\Delta b_{22}$ in that they enter with different signs but similar magnitude, thus leading to increased uncertainty in the final result for $\Delta b_{5}$. In spite of those delicate cancellations, we are able to resolve the fifth-order contribution, as shown already in Fig. 1(b). Nevertheless, the size of the error bars of $\Delta b_{32}$ is larger than that of $\Delta b_{41}$. This may come as a surprise given the results of Ref. [41], whose uncertainty at $\beta \omega=0$ for $\Delta b_{41}$ is larger than for $\Delta b_{32}$. Those results were calculated at the same $N_{\tau}=9$ order for both coefficients, using an analytic cancellation of volume-dependent terms. In contrast, in this work we achieved $N_{\tau}=12$ for $\Delta b_{41}$ but only $N_{\tau}=8$ for $\Delta b_{32}$, due to the increasing computational cost of canceling the volume-dependent terms, which is done numerically in the trapped case.

\section{Applications to thermodynamics}

Having obtained the precise form of $\Delta b_{3}, \Delta b_{4}$, and $\Delta b_{5}$ as functions of $\beta \omega$ for harmonically trapped fermions in the unitary limit, we apply those results to obtain thermodynamic information. As an example, we report here the compressibility and magnetic susceptibility, respectively $\chi_{+}$and $\chi_{-}$, defined as

$$
\chi_{ \pm}=\frac{1}{\beta} \frac{\partial^{2} \ln \mathcal{Z}}{\partial h_{ \pm}^{2}}
$$



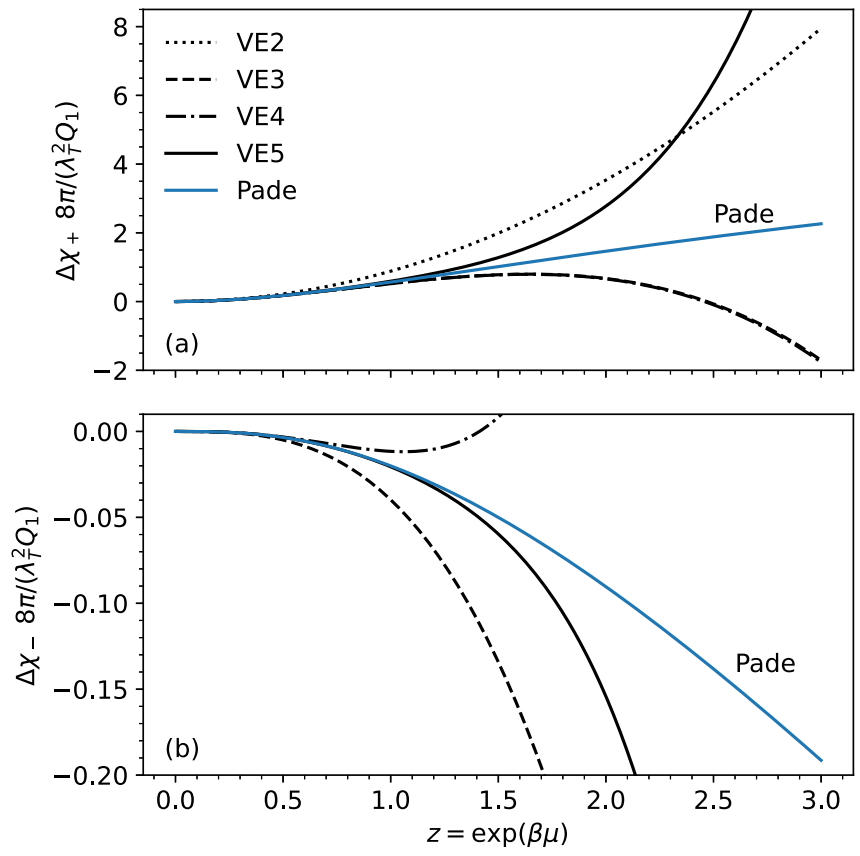

FIG. 3. (a) Interaction effects on the compressibility $\Delta \chi_{+}$, in units of $8 \pi /\left(\lambda_{T}^{2} Q_{1}\right)$, as a function of the fugacity $z$ for a harmonically trapped unitary Fermi gas at $\beta \omega=1$. The second, third, fourth, and fifth-order VE results are shown, respectively, with dotted, dashed, dashed-dotted, and solid lines. The Padé resummed result (with a [3/2] approximant) is shown as a blue line. (b) Interaction effects on the magnetic susceptibility $\Delta \chi_{-}$as a function of $z$, for the same parameters as in (a). The second-order VE is omitted because it is identically zero for $\Delta \chi_{-}$.

where $h_{ \pm}=\left(\mu_{\uparrow} \pm \mu_{\downarrow}\right) / 2$ and $\mu_{s}$ is the chemical potential for spin- $s$ particles. The interaction effects on $\chi_{ \pm}$are

$$
\Delta \chi_{ \pm}=\frac{\lambda_{T}^{2}}{8 \pi} Q_{1} \sum_{n=3}^{\infty} \sum_{m+j=n}(m \pm j)^{2} \Delta b_{m j} z_{\uparrow}^{m} z_{\downarrow}^{j},
$$

where $z_{s}=e^{\beta \mu_{s}}$ is the fugacity for spin-s particles. Our results, shown in Fig. 3, indicate that the partial sums of the VE display large variations for $\Delta \chi_{ \pm}$as the VE order is increased, in particular for $z \geqslant 1$. However, we also see that, using the high-order coefficients we calculated here, it is possible to carry out a Padé resummation [and related strategies (see, e.g., [48])] to obtain sensible results for static response functions even as far as $z=3$.

\section{CONCLUSION AND OUTLOOK}

In this work we have determined the frequency dependence of the virial coefficients $b_{n}$ of harmonically trapped spin- $\frac{1}{2}$ fermions at unitarity. We used a discretization of the imaginary-time direction and a Suzuki-Trotter factorization of the transfer matrix, together with automated algebra methods, to calculate canonical partition functions and from them the interaction-induced change $\Delta b_{n}$, for $n=3,4,5$, which we extrapolated to the continuous-time limit. To complement those numerical results, we provided analytic formulas for $\Delta b_{n}$ in coarse lattices for arbitrary trap frequency and spatial dimension. Using our final $\Delta b_{n}$, we calculate the compressibility and susceptibility of the unitary Fermi gas and showed that the VE can be Padé resummed to obtain sensible results even as far as $z=3$.

On the technical side, the above calculations were enabled by generalizing our former free-space formalism and automation, developed in momentum space, to the present trapped case, by making extensive use of the Mehler kernel and automated evaluation of Gaussian integrals using determinants. The resulting approach presents a fundamental difference with its free-space counterpart: the volume cancellations among canonical partition functions must be resolved numerically, which is crucial in order to access the $\Delta b_{n}$ as these are intensive quantities.

Our coordinate-space implementation could be generalized further to other geometries such as highly deformed harmonic traps, which could in turn be used to explore the dimensional crossover of the virial expansion. Nonharmonic potentials can also be addressed, as long as the single-particle kernel $\rho(\mathbf{x}, \mathbf{y})$ is known analytically and can be expanded in a basis of Gaussian functions. Additionally, more complicated interactions, such as those appearing in nuclear physics, could be implemented by expanding the matrix elements of $e^{-\tau \hat{V}}$ in terms of Gaussians, such that the resulting calculations can always be reduced to sums of determinants.

\section{ACKNOWLEDGMENT}

This material is based upon work supported by the National Science Foundation under Grants No. PHY1452635 and No. PHY2013078.

\section{APPENDIX A: TRANSFER MATRICES}

In the main text we showed the form of the Suzuki-Trotter factorized transfer matrix $\mathcal{M}_{11}$ of the $1+1$ subspace, written in the coordinate representation. We show here how to construct that matrix in more detail and present results for its higher-body counterparts. We will only consider here the subspaces where the interaction plays a role, as the noninteracting subspaces (e.g., those with $N+0$ particles) are trivial.

We begin by defining the product coordinate and harmonic oscillator states for two distinguishable particles in $d$ spatial dimensions

$$
\begin{aligned}
& \left.\mid \mathbf{x}_{1} \mathbf{x}_{2}\right)=\left|\mathbf{x}_{1}\right\rangle\left|\mathbf{x}_{2}\right\rangle, \\
& \left.\mid \mathbf{n}_{1} \mathbf{n}_{2}\right)=\left|\mathbf{n}_{1}\right\rangle\left|\mathbf{n}_{2}\right\rangle .
\end{aligned}
$$

Using these states, we have

$$
\left.\left.\exp (-\tau \hat{V}) \mid \mathbf{x}_{1} \mathbf{x}_{2}\right)=\left[\mathbb{1}+C \delta\left(\mathbf{x}_{1}-\mathbf{x}_{2}\right)\right] \mid \mathbf{x}_{1} \mathbf{x}_{2}\right),
$$

where $C=\left[\exp \left(\tau g / \ell^{d}\right)-1\right] \ell^{d}$, and so we obtain

$$
\begin{aligned}
{\left[\mathcal{M}_{11}\right]_{\mathbf{X}, \mathbf{Y}}=} & \sum_{\mathbf{n}_{1}, \mathbf{n}_{2}} e^{-\tau \epsilon_{2}\left(\mathbf{n}_{1}, \mathbf{n}_{2}\right)}\left(\mathbf{x}_{1} \mathbf{x}_{2} \mid \mathbf{n}_{1} \mathbf{n}_{2}\right)\left(\mathbf{n}_{1} \mathbf{n}_{2} \mid \mathbf{y}_{1} \mathbf{y}_{2}\right) \\
& \times\left[\mathbb{1}+C \delta\left(\mathbf{y}_{1}-\mathbf{y}_{2}\right)\right]
\end{aligned}
$$

where

$$
\epsilon_{2}\left(\mathbf{n}_{1}, \mathbf{n}_{2}\right)=\sum_{i=1}^{2} \epsilon\left(\mathbf{n}_{i}\right)
$$


and

$$
\epsilon(\mathbf{n})=\omega \sum_{j=1}^{d}\left([\mathbf{n}]_{j}+1 / 2\right)
$$

We can do the sum over $\mathbf{n}_{1}, \mathbf{n}_{2}$ using the Mehler kernel:

$\sum_{\mathbf{n}_{1}, \mathbf{n}_{2}} e^{-\tau \epsilon_{2}\left(\mathbf{n}_{1}, \mathbf{n}_{2}\right)}\left(\mathbf{x}_{1} \mathbf{x}_{2} \mid \mathbf{n}_{1} \mathbf{n}_{2}\right)\left(\mathbf{n}_{1} \mathbf{n}_{2} \mid \mathbf{y}_{1} \mathbf{y}_{2}\right)=\rho\left(\mathbf{x}_{1}, \mathbf{y}_{1}\right) \rho\left(\mathbf{x}_{2}, \mathbf{y}_{2}\right)$,

where the kernel $\rho(\mathbf{x}, \mathbf{y})$ is as shown in Eq. (21).

\section{Three-particle space}

Here we will need

$$
\left.\exp (-\tau \hat{V}) \mid \mathbf{X})=\left\{\mathbb{1}+C\left[\delta\left(\mathbf{x}_{1}-\mathbf{x}_{3}\right)+\delta\left(\mathbf{x}_{2}-\mathbf{x}_{3}\right)\right]\right\} \mid \mathbf{X}\right)
$$

where $\mathbf{X}=\left(\mathbf{x}_{1}, \mathbf{x}_{2}, \mathbf{x}_{3}\right)$ is a collective index, and we use $\left.\mid \mathbf{X}\right)$ to denote a state of distinguishable particles (i.e., no antisymmetrization among the $\mathbf{x}_{i}$ labels). Thus,

$$
\begin{aligned}
{\left[\mathcal{M}_{21}\right]_{\mathbf{X}, \mathbf{Y}}=} & \rho\left(\mathbf{x}_{1}, \mathbf{y}_{1}\right) \rho\left(\mathbf{x}_{2}, \mathbf{y}_{2}\right) \rho\left(\mathbf{x}_{3}, \mathbf{y}_{3}\right) \\
& \times\left\{\mathbb{1}+C\left[\delta\left(\mathbf{y}_{1}-\mathbf{y}_{3}\right)+\delta\left(\mathbf{y}_{2}-\mathbf{y}_{3}\right)\right]\right\} .
\end{aligned}
$$

\section{Four-particle space}

Here we distinguish the $3+1$ subspace from the $2+2$ subspace. For the $3+1$ case,

$$
\begin{aligned}
& \exp (-\tau \hat{V}) \mid \mathbf{X}) \\
& \left.\quad=\left\{\mathbb{1}+C\left[\delta\left(\mathbf{x}_{1}-\mathbf{x}_{4}\right)+\delta\left(\mathbf{x}_{2}-\mathbf{x}_{4}\right)+\delta\left(\mathbf{x}_{3}-\mathbf{x}_{4}\right)\right]\right\} \mid \mathbf{X}\right),
\end{aligned}
$$

where $\mathbf{X}=\left(\mathbf{x}_{1}, \mathbf{x}_{2}, \mathbf{x}_{3}, \mathbf{x}_{4}\right)$, and so we obtain

$$
\begin{aligned}
{\left[\mathcal{M}_{31}\right]_{\mathbf{X}, \mathbf{Y}}=} & \rho\left(\mathbf{x}_{1}, \mathbf{y}_{1}\right) \rho\left(\mathbf{x}_{2}, \mathbf{y}_{2}\right) \rho\left(\mathbf{x}_{3}, \mathbf{y}_{3}\right) \rho\left(\mathbf{x}_{4}, \mathbf{y}_{4}\right) \\
& \times\left\{\mathbb{1}+C\left[\delta\left(\mathbf{y}_{1}-\mathbf{y}_{4}\right)+\delta\left(\mathbf{y}_{2}-\mathbf{y}_{4}\right)+\delta\left(\mathbf{y}_{3}-\mathbf{y}_{4}\right)\right]\right\} .
\end{aligned}
$$

For the $2+2$ case, on the other hand,

$$
\begin{aligned}
\exp (-\tau \hat{V}) \mid \mathbf{X})= & \left\{\mathbb{1}+C\left[\delta\left(\mathbf{x}_{1}-\mathbf{x}_{3}\right)+\delta\left(\mathbf{x}_{1}-\mathbf{x}_{4}\right)\right.\right. \\
& \left.+\delta\left(\mathbf{x}_{2}-\mathbf{x}_{3}\right)+\delta\left(\mathbf{x}_{2}-\mathbf{x}_{4}\right)\right] \\
& +C^{2}\left[\delta\left(\mathbf{x}_{1}-\mathbf{x}_{3}\right) \delta\left(\mathbf{x}_{2}-\mathbf{x}_{4}\right)\right. \\
& \left.\left.\left.+\delta\left(\mathbf{x}_{1}-\mathbf{x}_{4}\right) \delta\left(\mathbf{x}_{2}-\mathbf{x}_{3}\right)\right]\right\} \mid \mathbf{X}\right),
\end{aligned}
$$

such that

$$
\begin{aligned}
& {\left[\mathcal{M}_{22}\right]_{\mathbf{X}, \mathbf{Y}}=\rho\left(\mathbf{x}_{1}, \mathbf{y}_{1}\right) \rho\left(\mathbf{x}_{2}, \mathbf{y}_{2}\right) \rho\left(\mathbf{x}_{3}, \mathbf{y}_{3}\right) \rho\left(\mathbf{x}_{4}, \mathbf{y}_{4}\right)} \\
& \quad \times\left\{\mathbb{1}+C\left[\delta\left(\mathbf{y}_{1}-\mathbf{y}_{3}\right)+\delta\left(\mathbf{y}_{1}-\mathbf{y}_{4}\right)\right.\right. \\
& \left.\quad+\delta\left(\mathbf{y}_{2}-\mathbf{y}_{3}\right)+\delta\left(\mathbf{y}_{2}-\mathbf{y}_{4}\right)\right]+C^{2}\left[\delta\left(\mathbf{y}_{1}-\mathbf{y}_{3}\right) \delta\left(\mathbf{y}_{2}-\mathbf{y}_{4}\right)\right. \\
& \left.\left.\quad+\delta\left(\mathbf{y}_{1}-\mathbf{y}_{4}\right) \delta\left(\mathbf{y}_{2}-\mathbf{y}_{3}\right)\right]\right\} .
\end{aligned}
$$

\section{Five-particle space}

Here we distinguish the $4+1$ subspace from the $3+2$ subspace. For the $4+1$ case,

$$
\left.\exp (-\tau \hat{V}) \mid \mathbf{X})=\left\{\mathbb{1}+C\left[\delta\left(\mathbf{x}_{1}-\mathbf{x}_{5}\right)+\delta\left(\mathbf{x}_{2}-\mathbf{x}_{5}\right)+\delta\left(\mathbf{x}_{3}-\mathbf{x}_{5}\right)+\delta\left(\mathbf{x}_{4}-\mathbf{x}_{5}\right)\right]\right\} \mid \mathbf{X}\right),
$$

where $\mathbf{X}=\left(\mathbf{x}_{1}, \mathbf{x}_{2}, \mathbf{x}_{3}, \mathbf{x}_{4}, \mathbf{x}_{5}\right)$, such that

$$
\begin{aligned}
{\left[\mathcal{M}_{41}\right]_{\mathbf{X}, \mathbf{Y}}=} & \rho\left(\mathbf{x}_{1}, \mathbf{y}_{1}\right) \rho\left(\mathbf{x}_{2}, \mathbf{y}_{2}\right) \rho\left(\mathbf{x}_{3}, \mathbf{y}_{3}\right) \rho\left(\mathbf{x}_{4}, \mathbf{y}_{4}\right) \rho\left(\mathbf{x}_{5}, \mathbf{y}_{5}\right) \\
& \times\left\{\mathbb{1}+C\left[\delta\left(\mathbf{y}_{1}-\mathbf{y}_{5}\right)+\delta\left(\mathbf{y}_{2}-\mathbf{y}_{5}\right)+\delta\left(\mathbf{y}_{3}-\mathbf{y}_{5}\right)+\delta\left(\mathbf{y}_{4}-\mathbf{y}_{5}\right)\right]\right\} .
\end{aligned}
$$

For the $3+2$ case, on the other hand,

$$
\begin{aligned}
\exp (-\tau \hat{V}) \mid \mathbf{X})= & \left\{\mathbb{1}+C\left[\delta\left(\mathbf{x}_{1}-\mathbf{x}_{4}\right)+\delta\left(\mathbf{x}_{1}-\mathbf{x}_{5}\right)+\delta\left(\mathbf{x}_{2}-\mathbf{x}_{4}\right)+\delta\left(\mathbf{x}_{2}-\mathbf{x}_{5}\right)+\delta\left(\mathbf{x}_{3}-\mathbf{x}_{4}\right)+\delta\left(\mathbf{x}_{3}-\mathbf{x}_{5}\right)\right]\right. \\
& +C^{2}\left[\delta\left(\mathbf{x}_{1}-\mathbf{x}_{4}\right) \delta\left(\mathbf{x}_{2}-\mathbf{x}_{5}\right)+\delta\left(\mathbf{x}_{1}-\mathbf{x}_{5}\right) \delta\left(\mathbf{x}_{2}-\mathbf{x}_{4}\right)+\delta\left(\mathbf{x}_{1}-\mathbf{x}_{4}\right) \delta\left(\mathbf{x}_{3}-\mathbf{x}_{5}\right)\right. \\
& \left.\left.\left.+\delta\left(\mathbf{x}_{1}-\mathbf{x}_{5}\right) \delta\left(\mathbf{x}_{3}-\mathbf{x}_{4}\right)\right]\right\} \mid \mathbf{X}\right),
\end{aligned}
$$

such that

$$
\begin{aligned}
& {\left[\mathcal{M}_{32}\right]_{\mathbf{X}, \mathbf{Y}}=\rho\left(\mathbf{x}_{1}, \mathbf{y}_{1}\right) \rho\left(\mathbf{x}_{2}, \mathbf{y}_{2}\right) \rho\left(\mathbf{x}_{3}, \mathbf{y}_{3}\right) \rho\left(\mathbf{x}_{4}, \mathbf{y}_{4}\right) \rho\left(\mathbf{x}_{5}, \mathbf{y}_{5}\right)} \\
& \quad \times\left\{\mathbb{1}+C\left[\delta\left(\mathbf{y}_{1}-\mathbf{y}_{4}\right)+\delta\left(\mathbf{y}_{1}-\mathbf{y}_{5}\right)+\delta\left(\mathbf{y}_{2}-\mathbf{y}_{4}\right)+\delta\left(\mathbf{y}_{2}-\mathbf{y}_{5}\right)+\delta\left(\mathbf{y}_{3}-\mathbf{y}_{4}\right)+\delta\left(\mathbf{y}_{3}-\mathbf{y}_{5}\right)\right]\right. \\
& \left.\quad+C^{2}\left[\delta\left(\mathbf{y}_{1}-\mathbf{y}_{4}\right) \delta\left(\mathbf{y}_{2}-\mathbf{y}_{5}\right)+\delta\left(\mathbf{y}_{1}-\mathbf{y}_{5}\right) \delta\left(\mathbf{y}_{2}-\mathbf{y}_{4}\right)+\delta\left(\mathbf{y}_{1}-\mathbf{y}_{4}\right) \delta\left(\mathbf{y}_{3}-\mathbf{y}_{5}\right)+\delta\left(\mathbf{y}_{1}-\mathbf{y}_{5}\right) \delta\left(\mathbf{y}_{3}-\mathbf{y}_{4}\right)\right]\right\}
\end{aligned}
$$

\section{APPENDIX B: ANALYTIC FORM OF VIRIAL COEFFICIENTS IN COARSE TEMPORAL LATTICES}

In this Appendix we complement the analytic expressions presented in the main text. For $N_{\tau}=1$, we find

$$
\Delta b_{41}=-\frac{2^{-d / 2} \Delta b_{2}}{\cosh ^{d / 2}(\beta \omega)[2 \cosh (\beta \omega)+2 \cosh (2 \beta \omega)+1]^{d / 2}}
$$

and

$$
\Delta b_{32}=-\Delta b_{2}\left[\frac{\cosh (\beta \omega)-1}{2 \cosh ^{2}(2 \beta \omega)-\cosh (\beta \omega)-1}\right]^{d / 2}
$$




$$
\begin{aligned}
& +2\left(\Delta b_{2}\right)^{2}\left\{\frac{1}{[1+2 \cosh (\beta \omega)+2 \cosh (2 \beta \omega)]^{d / 2}}+\frac{2^{d}}{[7+8 \cosh (2 \beta \omega)]^{d / 2}}\right. \\
& \left.-\frac{2^{d}}{[11+16 \cosh (\beta \omega)+8 \cosh (2 \beta \omega)]^{d / 2}}\right\} .
\end{aligned}
$$

For $N_{\tau}=2$, we find

$$
\begin{aligned}
& \Delta b_{11}=\frac{C}{\lambda_{T}^{d}}\left(\frac{\beta \omega}{\sinh (\beta \omega)}\right)^{d / 2} \frac{1}{2^{d / 2}}+\left(\frac{C}{\lambda_{T}^{d}}\right)^{2}\left(\frac{(\beta \omega)^{2}}{\sinh ^{2}(\beta \omega / 2)}\right)^{d / 2} \frac{1}{2^{d+1}}, \\
& \Delta b_{21}=\frac{C}{\lambda_{T}^{d}}\left(\frac{\beta \omega}{\sinh (\beta \omega)}\right)^{d / 2} \frac{-1}{[2+4 \cosh (\beta \omega)]^{d / 2}} \\
& +\left(\frac{C}{\lambda_{T}^{d}}\right)^{2}\left(\frac{(\beta \omega)^{2}}{\sinh ^{2}(\beta \omega / 2)}\right)^{d / 2}\left\{\frac{1}{2^{d+1}} \frac{1}{[1+2 \cosh (\beta \omega)]^{d / 2}}+\frac{-1}{[7+8 \cosh (\beta \omega)]^{d / 2}}\right\}, \\
& \Delta b_{31}=\frac{C}{\lambda_{T}^{d}}\left(\frac{\beta \omega}{\sinh (\beta \omega)}\right)^{d / 2} \frac{1}{2^{d}} \frac{1}{\{[1+\cosh (\beta \omega)+\cosh (2 \beta \omega)]\}^{d / 2}} \\
& +\left(\frac{C}{\lambda_{T}^{d}}\right)^{2}\left(\frac{(\beta \omega)^{2}}{\sinh ^{2}(\beta \omega / 2)}\right)^{d / 2}\left\{\frac{1}{[10+14 \cosh (\beta \omega)+8 \cosh (2 \beta \omega)]^{d / 2}}+\frac{-1}{4^{d}} \frac{1}{\left[2 \cosh ^{2}(\beta \omega / 2) \cosh (\beta \omega)\right]^{d / 2}}\right. \\
& \left.+\frac{1}{2^{2 d+1}} \frac{1}{\left\{\cosh ^{2}(\beta \omega / 2)[1+2 \cosh (\beta \omega)]\right\}^{d / 2}}\right\}, \\
& \Delta b_{22}=\frac{C}{\lambda_{T}^{d}}\left(\frac{\beta \omega}{\sinh (\beta \omega)}\right)^{d / 2} \frac{1}{4^{d}} \frac{1}{\left[\cosh ^{2}(\beta \omega / 2) \cosh (\beta \omega)\right]^{d / 2}} \\
& +\left(\frac{C}{\lambda_{T}^{d}}\right)^{2}\left(\frac{(\beta \omega)^{2}}{\sinh ^{2}(\beta \omega / 2)}\right)^{d / 2}\left\{\frac{1}{2^{3 d+1}} \frac{1}{\left[\cosh ^{4}(\beta \omega / 2)\right]^{d / 2}}+\frac{-1}{\left[32 \cosh ^{2}(\beta \omega / 2) \cosh (\beta \omega)\right]^{d / 2}}\right. \\
& \left.+\frac{2}{\left\{\cosh ^{2}(\beta \omega / 2)[16+32 \cosh (\beta \omega)]\right\}^{d / 2}}+\frac{-2}{[10+14 \cosh (\beta \omega)+8 \cosh (2 \beta \omega)]^{d / 2}}\right\} \\
& +\left(\frac{C}{\lambda_{T}^{d}}\right)^{3}\left(\frac{(\beta \omega)^{3}}{\sinh ^{3}(\beta \omega / 2)}\right)^{d / 2}\left\{\frac{-4}{[24 \cosh (\beta \omega / 2)+16 \cosh (3 \beta \omega / 2)]^{d / 2}}\right. \\
& \left.+\frac{2}{\left[64 \cosh ^{3}(\beta \omega / 2)\right]^{d / 2}}+\left[\frac{\sinh (\beta \omega / 2)}{8 \sinh (2 \beta \omega)}\right]^{d / 2}\right\} \\
& +\left(\frac{C}{\lambda_{T}^{d}}\right)^{4}\left(\frac{(\beta \omega)^{4}}{\sinh ^{4}(\beta \omega / 2)}\right)^{d / 2}\left\{\frac{-1}{[16+32 \cosh (\beta \omega)]^{d / 2}}+\frac{3}{4} \frac{1}{\left[64 \cosh ^{2}(\beta \omega / 2)\right]^{d / 2}}\right\}, \\
& \Delta b_{41}=\frac{C}{\lambda_{T}^{d}}\left(\frac{\beta \omega}{\sinh (\beta \omega)}\right)^{d / 2} \frac{-1}{\{4(1+2 \cosh [\beta \omega)+\cosh (2 \beta \omega)+\cosh (3 \beta \omega)]\}^{d / 2}} \\
& +\left(\frac{C}{\lambda_{T}^{d}}\right)^{2}\left(\frac{(\beta \omega)^{2}}{\sinh ^{2}(\beta \omega / 2)}\right)^{d / 2}\left\{\frac{-1}{[13+20 \cosh (\beta \omega)+14 \cosh (2 \beta \omega)+8 \cosh (3 \beta \omega)]^{d / 2}}\right. \\
& +\frac{1}{\left[4(3+6 \cosh (\beta \omega)+4 \cosh (2 \beta \omega)+2 \cosh (3 \beta \omega)]^{d / 2}\right.} \\
& +\frac{-1}{[23+40 \cosh (\beta \omega)+24 \cosh (2 \beta \omega)+8 \cosh (3 \beta \omega)]^{d / 2}} \\
& \left.+\frac{1}{2} \frac{1}{\left[4 \cosh ^{2}(\beta \omega / 2)(4+8 \cosh (\beta \omega)+8 \cosh (2 \beta \omega)]^{d / 2}\right.}\right\},
\end{aligned}
$$

$$
\begin{aligned}
\Delta b_{32}= & \frac{C}{\lambda_{T}^{d}}\left(\frac{\beta \omega}{\sinh (\beta \omega)}\right)^{d / 2} \frac{-1}{[6+12 \cosh (\beta \omega)+8 \cosh (2 \beta \omega)+4 \cosh (3 \beta \omega)]^{d / 2}} \\
& +\left(\frac{C}{\lambda_{T}^{d}}\right)^{2}\left(\frac{(\beta \omega)^{2}}{\sinh ^{2}(\beta \omega / 2)}\right)^{d / 2}\left\{\frac{1}{\left\{4 \cosh ^{2}(\beta \omega / 2)[4+8 \cosh (\beta \omega)+8 \cosh (2 \beta \omega)]\right\}^{d / 2}}\right.
\end{aligned}
$$




$$
\begin{aligned}
& +\frac{1}{\left\{\cosh ^{2}(\beta \omega / 2)[28+32 \cosh (2 \beta \omega)]\right\}^{d / 2}}+\frac{-1}{\left\{\cosh ^{2}(\beta \omega / 2)[44+64 \cosh (\beta \omega)+32 \cosh (2 \beta \omega)]\right\}^{d / 2}} \\
& +\frac{-2}{[23+40 \cosh (\beta \omega)+24 \cosh (2 \beta \omega)+8 \cosh (3 \beta \omega)]^{d / 2}}+\frac{1}{\left\{\cosh ^{2}(\beta \omega)[28+32 \cosh (\beta \omega)]\right\}^{d / 2}} \\
& +\frac{2}{\left\{8 \cosh ^{2}(\beta \omega / 2)[3+3 \cosh (\beta \omega)+4 \cosh (2 \beta \omega)]\right\}^{d / 2}}+\frac{-1}{\left\{[1+2 \cosh (\beta \omega)]^{2}[7+8 \cosh (\beta \omega)]\right\}^{d / 2}} \\
& \left.+\frac{1}{2} \frac{1}{\{4(3+6 \cosh (\beta \omega)+4 \cosh [2 \beta \omega)+2 \cosh (3 \beta \omega)]\}^{d / 2}}\right\} \\
& +\left(\frac{C}{\lambda_{T}^{d}}\right)^{3}\left(\frac{(\beta \omega)^{3}}{\sinh ^{3}(\beta \omega / 2)}\right)^{d / 2}\left\{\frac{1}{\{\cosh (\beta \omega)[8+32 \cosh (\beta \omega)]\}^{d / 2}}\left[\frac{\sinh (\beta \omega / 2)}{\sinh (\beta \omega)}\right]^{d / 2}\right. \\
& +\frac{-2}{\{76 \cosh (\beta \omega / 2)+16[3 \cosh (3 \beta \omega / 2)+\cosh (5 \beta \omega / 2)]\}^{d / 2}}+\frac{-2}{\{16 \cosh (\beta \omega / 2)[1+2 \cosh (\beta \omega)+2 \cosh (2 \beta \omega)]\}^{d / 2}} \\
& +\frac{2}{\{4 \cosh (\beta \omega / 2)[5+12 \cosh (\beta \omega)+8 \cosh (2 \beta \omega)]\}^{d / 2}}+\frac{6}{\{8 \cosh (\beta \omega / 2) \cosh (\beta \omega)[7+8 \cosh (\beta \omega)]\}^{d / 2}} \\
& \left.+\frac{-4}{\{4 \cosh (\beta \omega / 2)[15+22 \cosh (\beta \omega)+8 \cosh (2 \beta \omega)]\}^{d / 2}}+\frac{1}{\left\{32 \cosh ^{3}(\beta \omega / 2)[1+4 \cosh (\beta \omega)]\right\}^{d / 2}}\right\} \\
& +\left(\frac{C}{\lambda_{T}^{d}}\right)^{4}\left(\frac{(\beta \omega)^{4}}{\sinh ^{4}(\beta \omega / 2)}\right)^{d / 2}\left\{\frac{-3}{[60+88 \cosh (\beta \omega)+32 \cosh (2 \beta \omega)]^{d / 2}}\right. \\
& +\frac{3}{[41+72 \cosh (\beta \omega)+32 \cosh (2 \beta \omega)]^{d / 2}}+\frac{1}{[33+40 \cosh (\beta \omega)+32 \cosh (2 \beta \omega)]^{d / 2}} \\
& +\frac{1}{2} \frac{1}{\{16[1+2 \cosh (\beta \omega)+2 \cosh (2 \beta \omega)]\}^{d / 2}}+\frac{-1}{\left\{4[1+4 \cosh (\beta \omega)]^{2}\right\}^{d / 2}} \\
& +\frac{-2}{[20+48 \cosh (\beta \omega)+32 \cosh (2 \beta \omega)]^{d / 2}}+\frac{2}{\left\{32 \cosh ^{2}[\beta \omega / 2)(1+4 \cosh (\beta \omega)]\right\}^{d / 2}} \\
& \left.+\frac{-1}{2} \frac{1}{\left\{[7+8 \cosh (\beta \omega)]^{2}\right\}^{d / 2}}+\frac{1}{2} \frac{1}{[25+48 \cosh (\beta \omega)+32 \cosh (2 \beta \omega)]^{d / 2}}\right\} .
\end{aligned}
$$

[1] P. Pieri, L. Pisani, and G. C. Strinati, BCS-BEC crossover at finite temperature in the broken-symmetry phase, Phys. Rev. B 70, 094508 (2004).

[2] A. Sedrakian, J. Mur-Petit, A. Polls, and H. Müther, Pairing in a two-component ultracold fermi gas: Phases with broken-space symmetries, Phys. Rev. A 72, 013613 (2005).

[3] C. Lobo, A. Recati, S. Giorgini, and S. Stringari, Normal State of a Polarized Fermi Gas at Unitarity, Phys. Rev. Lett. 97, 200403 (2006).

[4] M. Randeria, Pre-pairing for condensation, Nat. Phys. 6, 561 (2010).

[5] J. P. Gaebler, J. T. Stewart, T. E. Drake, D. S. Jin, A. Perali, P. Pieri, and G. C. Strinati, Observation of pseudogap behaviour in a strongly interacting Fermi gas, Nat. Phys. 6, 569 (2010).

[6] Q. Chen and J. Wang, Pseudogap phenomena in ultracold atomic fermi gases, Front. Phys. 9, 539 (2014).

[7] E. J. Mueller, Review of pseudogaps in strongly interacting fermi gases, Rep. Prog. Phys. 80, 104401 (2017).

[8] S. Jensen, C. N. Gilbreth, and Y. Alhassid, The pseudogap regime in the unitary fermi gas, Eur. Phys. J.: Spec. Top. 227, 2241 (2019).
[9] A. Richie-Halford, J. E. Drut, and A. Bulgac, Emergence of a Pseudogap in the bcs-bec Crossover, Phys. Rev. Lett. 125, 060403 (2020).

[10] L. Rammelmüller, Y. Hou, J. E. Drut, and J. Braun, Pairing and the spin susceptibility of the polarized unitary fermi gas in the normal phase, Phys. Rev. A 103, 043330 (2021).

[11] R. Pathria and P. D. Beale, Statistical Mechanics (Third Edition), edited by R. Pathria and P. D. Beale (Academic, Boston, 2011).

[12] X.-J. Liu, Virial expansion for a strongly correlated fermi system and its application to ultracold atomic fermi gases, Phys. Rep. 524, 37 (2013).

[13] E. Beth and G. E. Uhlenbeck, The quantum theory of the non-ideal gas. ii. behaviour at low temperatures, Physica (Amsterdam) 4, 915 (1937).

[14] Proceedings of the International School of Physics "Enrico Fermi”, Course CLXIV, Varenna, edited by M. Inguscio, W. Ketterle, and C. Salomon (IOS, Amsterdam, 2007).

[15] The BCS-BEC Crossover and the Unitary Fermi Gas, edited by W. Zwerger (Springer, Berlin, 2012). 
[16] T.-L. Ho, Universal Thermodynamics of Degenerate Quantum Gases in the Unitarity Limit, Phys. Rev. Lett. 92, 090402 (2004).

[17] E. Braaten and H.-W. Hammer, Universality in few-body systems with large scattering length, Phys. Rep. 428, 259 (2006).

[18] I. Bloch, J. Dalibard, and W. Zwerger, Many-body physics with ultracold gases, Rev. Mod. Phys. 80, 885 (2008).

[19] S. Giorgini, L. P. Pitaevskii, and S. Stringari, Theory of ultracold atomic fermi gases, Rev. Mod. Phys. 80, 1215 (2008).

[20] J. Levinsen, P. Massignan, S. Endo, and M. M. Parish, Universality of the unitary fermi gas: a few-body perspective, J. Phys. B: At., Mol. Opt. Phys. 50, 072001 (2017).

[21] G. C. Strinati, P. Pieri, G. Röpke, P. Schuck, and M. Urban, The bcs-bec crossover: From ultra-cold fermi gases to nuclear systems, Phys. Rep. 738, 1 (2018).

[22] X.-J. Liu, H. Hu, and P. D. Drummond, Virial Expansion for a Strongly Correlated Fermi Gas, Phys. Rev. Lett. 102, 160401 (2009).

[23] X.-J. Liu, H. Hu, and P. D. Drummond, Three attractively interacting fermions in a harmonic trap: Exact solution, ferromagnetism, and high-temperature thermodynamics, Phys. Rev. A 82, 023619 (2010).

[24] D. B. Kaplan and S. Sun, New Field-Theoretic Method for the Virial Expansion, Phys. Rev. Lett. 107, 030601 (2011).

[25] C. Gao, S. Endo, and Y. Castin, The third virial coefficient of a two-component unitary fermi gas across an efimov-effect threshold, Europhys. Lett. 109, 16003 (2015).

[26] S. Endo and Y. Castin, The interaction-sensitive states of a trapped two-component ideal fermi gas and application to the virial expansion of the unitary Fermi gas, J. Phys. A: Math. Theor. 49, 265301 (2016).

[27] X. Leyronas, Virial expansion with Feynman diagrams, Phys. Rev. A 84, 053633 (2011).

[28] Y. Castin and F. Werner, Troisieme coefficient du viriel du gaz de bose unitaire, Can. J. Phys. 91, 382 (2013).

[29] Y. Yan and D. Blume, Path-Integral Monte Carlo Determination of the Fourth-Order Virial Coefficient for a Unitary TwoComponent Fermi Gas with Zero-Range Interactions, Phys. Rev. Lett. 116, 230401 (2016).

[30] D. Rakshit, K. M. Daily, and D. Blume, Natural and unnatural parity states of small trapped equal-mass two-component fermi gases at unitarity and fourth-order virial coefficient, Phys. Rev. A 85, 033634 (2012).

[31] V. Ngampruetikorn, M. M. Parish, and J. Levinsen, Hightemperature limit of the resonant fermi gas, Phys. Rev. A 91, 013606 (2015).

[32] S. Endo and Y. Castin, Absence of a four-body efimov effect in the $2+2$ fermionic problem, Phys. Rev. A 92, 053624 (2015).

[33] S. E. Gharashi, K. M. Daily, and D. Blume, Three $s$-waveinteracting fermions under anisotropic harmonic confinement:
Dimensional crossover of energetics and virial coefficients, Phys. Rev. A 86, 042702 (2012).

[34] S.-G. Peng, S.-H. Zhao, and K. Jiang, Virial expansion of a harmonically trapped fermi gas across a narrow feshbach resonance, Phys. Rev. A 89, 013603 (2014).

[35] T. Kristensen, X. Leyronas, and L. Pricoupenko, Second-order virial expansion for an atomic gas in a harmonic waveguide, Phys. Rev. A 93, 063636 (2016).

[36] K. J. Morrell, C. E. Berger, and J. E. Drut, Third- and fourthorder virial coefficients of harmonically trapped fermions in a semiclassical approximation, Phys. Rev. A 100, 063626 (2019).

[37] C. R. Shill and J. E. Drut, Virial coefficients of one-dimensional and two-dimensional fermi gases by stochastic methods and a semiclassical lattice approximation, Phys. Rev. A 98, 053615 (2018).

[38] Y. Hou, A. J. Czejdo, J. DeChant, C. R. Shill, and J. E. Drut, Leading- and next-to-leading-order semiclassical approximation to the first seven virial coefficients of spin-1/2 fermions across spatial dimensions, Phys. Rev. A 100, 063627 (2019).

[39] C. E. Berger, K. J. Morrell, and J. E. Drut, Thermodynamics of rotating quantum matter in the virial expansion, Phys. Rev. A 102, 023309 (2020).

[40] A. J. Czejdo, J. E. Drut, Y. Hou, J. R. McKenney, and K. J. Morrell, Virial coefficients of trapped and untrapped threecomponent fermions with three-body forces in arbitrary spatial dimensions, Phys. Rev. A 101, 063630 (2020).

[41] Y. Hou and J. E. Drut, Fourth- and Fifth-Order Virial Coefficients from Weak Coupling to Unitarity, Phys. Rev. Lett. 125, 050403 (2020).

[42] Y. Hou and J. E. Drut, Virial expansion of attractively interacting fermi gases in one, two, and three dimensions, up to fifth order, Phys. Rev. A 102, 033319 (2020).

[43] M. J. Ku, A. T. Sommer, L. W. Cheuk, and M. W. Zwierlein, Revealing the superfluid lambda transition in the universal thermodynamics of a unitary fermi gas, Science 335, 563 (2012).

[44] S. Nascimbène, N. Navon, K. Jiang, F. Chevy, and C. Salomon, Exploring the thermodynamics of a universal fermi gas, Nature (London) 463, 1057 (2010).

[45] T. D. Lee and C. N. Yang, Many-body problem in quantum statistical mechanics. i. general formulation, Phys. Rev. 113, 1165 (1959).

[46] T. D. Lee and C. N. Yang, Many-body problem in quantum statistical mechanics. ii. virial expansion for hard-sphere gas, Phys. Rev. 116, 25 (1959).

[47] J. McCabe and S. Ouvry, Perturbative three-body spectrum and the third virial coefficient in the anyon model, Phys. Lett. B 260, 113 (1991).

[48] R. Rossi, T. Ohgoe, K. Van Houcke, and F. Werner, Resummation of Diagrammatic Series with Zero Convergence Radius for Strongly Correlated Fermions, Phys. Rev. Lett. 121, 130405 (2018). 